\title{
Applications of radiofrequency ablation in the treatment of breast cancer
}

\author{
S. E. Singletary \\ Department of Surgical Oncology, The University of Texas MD Anderson Cancer Center, Houston, TX, USA.
}

\begin{abstract}
Physicians are testing minimally invasive ablation techniques to determine if they will be acceptable substitutes for surgical removal of primary breast tumors. In radiofrequency ablation (RFA), frictional heating of the tissue is caused by the rapid movement of ions attempting to follow a high frequency alternating current moving between two electrodes. Localization of a small electrode in the tumor is accomplished under ultrasound guidance, and a larger electrode pad is placed on the outside of the body. Five pilot studies have demonstrated that RFA can effectively ablate tumors up to $3 \mathrm{~cm}$ in size. In addition to treating small primary tumors, RFA may also be useful for consolidation therapy after lumpectomy, for treatment of inoperable tumors in endstage patients, for treatment of locally advanced disease as a substitute for neoadjuvant chemotherapy, and for treatment of breast cancer metastases, such as bone, liver, and kidney. Clinical trials are needed to assess treatment outcomes and to monitor the long-term fate of ablated tissue.
\end{abstract}

Keywords: Minimally invasive techniques; Primary breast cancer; Radiofrequency ablation

\section{Introduction}

As recently as 30 years ago, the treatment standard for almost all cases of breast cancer was mastectomy combined with axillary lymph node dissection. Since then, however, a number of developments have resulted in a trend towards less invasive surgery. The widespread use of screening mammography has meant that, on average, breast tumors are now much smaller when first discovered, with a lower probability of nodal involvement. Large-scale clinical trials have shown that breast conservation therapy, involving lumpectomy and radiation therapy, results in survival rates equivalent to those seen with mastectomy. The demonstration that survival is not related to the

Correspondence to: S. Eva Singletary, Department of Surgical Oncology, The University of Texas MD Anderson Cancer Center, 1515 Holcombe Boulevard, Box 444, Houston, TX 77030-4095, USA. E-mail: esinglet@mdanderson.org; Tel: +1713 792 6937; Fax: +1713 7922225

Received 14/06/05 Revised 23/06/05

Accepted 23/06/05

First published online 30/09/05

BCO/374/2005/FO extent of surgery reflects the fact that breast cancer is a systemic disease, usually requiring systemic therapy.

Following this trend, clinicians are now considering the possibility of even less invasive approaches for local treatment of the primary tumor. Ablation techniques which either heat or cool tumor cells sufficiently to kill them are being tested to determine if they will be acceptable substitutes for surgical removal of the tumor. For breast cancer, one of the most promising of these techniques is radiofrequency ablation (RFA).

\section{RFA technology}

RFA is produced from high-frequency alternating current moving between a small electrode placed in the tumor and a larger electrode pad placed on the skin surface, usually on the hip or thigh. Frictional heating of the tissue around the small electrode is caused by the rapid movement of ions in the tissue attempting to follow the alternating current. The size of the tumor that can be ablated is a function of the size and shape of the electrode. Most practitioners use a device in 
which secondary electrodes can be deployed from the tip in a star-like array, yielding an ablation zone of $3-5 \mathrm{~cm}$ in diameter.

\section{The use of imaging}

RFA uses imaging technology to localize the tumor, to determine if it is the appropriate size and in an appropriate location for RFA, to guide the placement of the probe, and (ideally) to monitor the course of RFA treatment. In most studies to date, ultrasonography (US) has been used to support RFA, but it has limitations. It is optimally effective only in small, regularly shaped tumors with clear borders, and it is not useful for visualizing the ablation process in real time. A recent study by Burak et al. [1] has found that MRI can be used with some success to monitor the course of treatment. In addition, MRI may be more accurate than US in visualizing the extent of disease and in detecting multicentric or multifocal tumors. However, MRI suffers from low specificity in discriminating between benign and malignant tumors, and is less useful for detecting ductal carcinoma in situ (DCIS) or lobular cancers.

\section{How successful has RFA been in the treatment of primary breast tumors?}

Five pilot studies have looked at the effectiveness of RFA in treating primary breast tumors (Table 1) [1-5]. Based on findings from the initial study by Jeffrey et al. in 1999 [2], subsequent studies restricted the use of RFA to patients with small tumors $(0.7-3.0 \mathrm{~cm}$ in diameter). In all of these studies, the tumor site was surgically removed after RFA and the tissue examined histologically for evidence of residual tumor.

In general, RFA ablation of the area targeted by US was successful in at least $90 \%$ of patients with small primary tumors, with minimal side effects. However, histologic examination revealed that, in some instances, US had underestimated the size of the tumor, with the result that residual tumor cells remained at the tumor site.

\section{Limitations in the use of RFA}

The success of RFA depends on patient selection and accurate imaging. Tumors should be small, with borders clearly visible on US. RFA is currently contraindicated in women with evidence of multifocal or multicentric tumors, or in women with biopsy-proven evidence of extensive DCIS or lobular cancer. In order to allow full deployment of the electrode tips and to avoid skin burns, the tumor should be located at least $1 \mathrm{~cm}$ from the chest wall and from the surface of the breast. Several studies have demonstrated that prior treatment with neoadjuvant chemotherapy reduces the accuracy of US assessment of tumor size $[6,7]$. Thus patients who have received presurgical chemotherapy are not good candidates for RFA.

\section{The future of RFA}

\section{New directions in tumor imaging}

As successful RFA is so heavily dependent on accurate imaging, new developments in imaging technology will be important. An example of this is positron emission tomography (PET), which produces images based on metabolic and physiologic functions occurring in living cells. A positron-emitting radionuclide is attached to a molecule (typically glucose or fluorodeoxyglucose (FDG)) that is taken up and metabolized at a high rate in tumor cells. A downside to using PET as a stand-alone technology is that there are almost no anatomic landmarks seen on PET scans. This is the opposite to that seen with CT scans, which offer high resolution anatomic imaging with appropriate contrast agents, but provide poor contrast between neoplastic and normal tissue. The development of integrated PET/CT scans combines the advantages of both technologies, and offers improved sensitivity and specificity compared to PET alone [8].

Fluorescence spectroscopy may be able to provide real-time assessment of lesions before treatment and of tumor margins after treatment. This technique is based on the observation that endogenous

Table 1. The use of RFA for the treatment of primary breast cancer: results of five pilot studies.

\begin{tabular}{llll}
\hline Reference & $\begin{array}{l}\text { No. of } \\
\text { patients }\end{array}$ & $\begin{array}{l}\text { Tumor size } \\
\text { (cm) }\end{array}$ & $\begin{array}{l}\text { \% of patients with complete } \\
\text { ablation of targeted zone }\end{array}$ \\
\hline Jeffrey et al. (1999) [2] & 5 & $4-7$ & $80 \%(4 / 5)$ \\
Izzo et al. (2001) [3] & 26 & $<3$ & $96 \%(25 / 26)$ \\
Mirza et al. (2002) [4] & 30 & $\leqslant 2$ & $93 \%(28 / 30)^{\mathrm{a}}$ \\
Burak et al. (2003) [1] & 10 & $<1.6$ & $90 \%(9 / 10)$ \\
Hayashi et al. (2003) [5] & 22 & $<2$ & $91 \%(20 / 22)^{\mathrm{b}}$ \\
\hline
\end{tabular}

a In 2 of the 28 patients with complete ablation of the targeted area, tumor size was underestimated by ultrasound.

${ }^{b}$ In one of the 20 patients with complete ablation of the targeted area, tumor size was underestimated by ultrasound. 
fluorophores (e.g. aromatic amino acids, structural proteins such as collagen and elastin) emit light that differs in concentration in benign vs. malignant tissue. The light can be detected using a fiberoptic probe that is delivered to the tumor site with a transdermal needle placed under US guidance. Breslin et al. [9] used fluorescence spectroscopy to examine breast tissue specimens from 32 patients with invasive breast cancer, and were able to distinguish benign from malignant tissue with a specificity of $92 \%$ and a sensitivity of $70 \%$.

\section{Combining RFA with other local treatments}

Studies to date have examined the usefulness of RFA for the treatment of small breast tumors, with the idea that this noninvasive treatment may be developed into a stand-alone outpatient procedure. However, RFA may be most useful when performed in combination with other local treatment.

In breast conservation therapy, radiation therapy is used in combination with lumpectomy to reduce the probability of local recurrence. It seems likely that this would also be true for RFA, especially since there is no opportunity to examine tumor margins after ablative therapy, and the possibility of residual tumor cells cannot be ruled out histologically. In a recent study, Horkan et al. [10] used a rat model of breast cancer to determine if radiation treatment used in combination with RFA would improve local control compared with RFA alone. They found that RFA in combination with radiation (total dose of $20 \mathrm{~Gy}$ ) resulted in complete local control in $92 \%$ of animals, compared with $9 \%$ of animals treated with RFA alone.

RFA might also be used as consolidation therapy after lumpectomy. In this case, occult residual tumor cells remaining in the surgical cavity would be treated with RFA, potentially making standard radiation therapy unnecessary.

\section{Non-curative uses of RFA}

RFA may also be valuable for the treatment of more advanced cancer. Fujimoto et al. [11] proposed that RFA might be used in place of neoadjuvant

Table 2. Potential uses for RFA in the treatment of breast cancer.

- Local treatment of small $(<3 \mathrm{~cm})$ tumors, with or without radiation therapy

- Consolidation therapy after lumpectomy

- Treatment of inoperable tumors in end-stage patients for symptomatic relief

- Substitute for neoadjuvant chemotherapy in patients with locally advanced breast cancer

- Treatment of breast cancer metastases to the bone, liver, kidney chemotherapy in treating patients with locally advanced breast cancer, avoiding the often severe side effects associated with that therapy. Vansonnenberg et al. [12] used RFA for the palliative treatment of a fungating and protuberant lesion occurring in the reconstructed breast of women with recurrent breast cancer. The RFA treatment resolved the spontaneous bleeding and oozing from the tumor, significantly reduced the size of the exophytic portion, and resulted in relief of discomfort in the patient.

RFA has been used successfully for the treatment of benign osteomas, and early studies indicate that it might also be effective for the treatment of breast cancer metastases to the bone $[13,14]$. Standard treatments for bone metastases include chemotherapy and radiation therapy, but some patients are unresponsive to these therapies. Even when successful, it may take as long as a month for patients to achieve any relief of pain. RFA may also be effective in the treatment of breast cancer metastases to other organs, including the liver.

\section{Conclusions}

The use of RFA is still at a developmental stage, with researchers exploring ways in which this technology will be most valuable (Table 2). Many questions still need to be answered: How will survival outcomes in early-stage patients treated with RFA with or without radiation therapy compare with those in patients treated with standard breast conservation therapy? What is the long-term fate of the ablated area after RFA? Will the necrosis and scarring be resorbed over time, or will it remain, interfering with future imaging studies? Could RFA prolong survival and/or increase quality of life in patients with metastatic disease who might otherwise be poor candidates for surgery? Clinical trials will be needed to provide answers to these questions, and determine if local ablative therapies like RFA will really be the next step forward in the management of breast cancer.

\section{References}

1. Burak Jr WE, Agnese DM, Povoski SP, et al. Radiofrequency ablation of invasive breast carcinoma followed by delayed surgical excision. Cancer 2003; 98: 1369-1376.

2. Jeffrey SS, Birdwell RL, Ikeda DM, et al. Radiofrequency ablation of breast cancer. Arch Surg 1999; 134: 1064-1068.

3. Izzo F, Thomas R, Delrio P, et al. Radiofrequency ablation in patients with primary breast carcinoma: a pilot study in 26 patients. Cancer 2001; 92: 2036-2044.

4. Mirza AN, Fornage BD, Sneige N, et al. Radiofrequency Ablation of Early-Stage Invasive Breast Tumors: Results of a Multi-Center Study. Proceedings of the 25th San Antonio Breast Cancer Symposium, San Antonio, Texas, December 2002, 450. 
5. Hayashi AH, Silver SF, van der Westhuizen NG, et al. Treatment of invasive breast carcinoma with ultrasoundguided radiofrequency ablation. Am J Surg 2003; 185: 429-435.

6. Chagpar AB, Middleton LP, Sahin AA, et al. Accuracy of physical examination, ultrasonography and mammography in predicting residual pathological tumor size in patients treated with neoadjuvant chemotherapy. Ann Surg 2005 (e-publication).

7. Akashi-Tanaka S, Fukutomi T, Watanabe $\mathrm{T}$, et al. Accuracy of contrast-enhanced computed tomography in the prediction of residual breast cancer after neoadjuvant chemotherapy. Int J Cancer 2001; 96: 66-73.

8. Hany TF, Steinert HC, Goerres GW, Buck A, von Schulthess GK. Improvement of diagnostic accuracy of PET imaging using a high performance in-line PET-CT system: preliminary results. Radiology 2002; 225: 575-581.

9. Breslin TM, Xu F, Palmer GM, Zhu C, Gilchrest KW, Ramanujam N. Autofluorescence and diffuse reflectance properties of malignant and benign breast tissues. Ann Surg Oncol 2004; 11: 65-70.

10. Horkan C, Dalal K, Coderre JA, et al. Reduced tumor growth with combined radiofrequency ablation and radiation therapy in a rat breast tumor model. Radiology 2005; 235: 81-88.

11. Fujimoto S, Kobayashi K, Takahashi M, et al. Clinical pilot studies on preoperative hyperthermic tumor ablation for advanced breast carcinoma using an $8 \mathrm{MHz}$ radiofrequency heating device. Int $J$ Hyperthermia 2003; 19: 13-22.

12. Vansonnenberg E, Shankar S, Parker L, et al. Palliative radiofrequency ablation of a fungating, symptomatic breast lesion. Am J Roentgenol 2005; 184: S126-S128.

13. Posteraro AF, Dupuy DE, Mayo-Smith WW. Radiofrequency ablation of bony metastatic disease. Clin Radiol 2004; 59: 803-811.

14. Patti JW, Neeman Z, Wood BJ. Radiofrequency ablation for cancer-associated pain. J Pain 2002; 3: 471-473. 